\title{
Prevalence of non-acute ischaemic cardiomyopathy in a Pakistani cohort
}

\author{
Muhammad Salman Ghazni, ${ }^{\oplus}$ Saba Aijaz, Rehan Malik, Asad Z Pathan
}

- Additional material is

published online only. To view please visit the journal online (http://dx.doi.org/10.1136/ heartasia-2018-011043).

Cardiology, Tabba Heart Institute, Karachi, Pakistan

\section{Correspondence to} Dr Asad Z Pathan, Cardiology, Tabba Heart Institute, Karachi 75950, Pakistan; asadzpathan@ gmail.com

Received 29 March 2018 Revised 8 January 2019 Accepted 9 January 2019

\section{Check for updates}

(C) Author(s) (or their employer(s)) 2019. No commercial re-use. See rights and permissions. Published by BMJ.

To cite: Ghazni MS, Aijaz S, Malik R, et al. Heart Asia 2019;11:e011043. doi:10.1136/

heartasia-2018-011043

\section{ABSTRACT}

Heart failure with reduced left ventricular ejection fraction (HFrEF) is a frequently encountered clinical scenario. Coronary angiography (CAG) is usually performed to assess obstructive epicardial coronary artery disease (CAD) and the resultant ischaemia as causes of HFrEF.

Objectives To determine the frequency of obstructive CAD (OCAD) in patients with HFrEF and its independent predictors and outcomes.

Methods Retrospective observational study in Tabba Heart Institute on patients who underwent CAG during the past 4 years. Patients with prior known CAD or revascularisation were excluded. OCAD was defined as per the criteria from Felker et al. Regression modelling was performed to evaluate the predictors of OCAD. Survival was compared between the groups using the log rank test.

Results Out of 2235 patients who underwent CAG, 260 had HFrEF as a primary indication for CAG and, of these, $119(45.8 \%)$ had OCAD. Major predictors of OCAD were age $>50$ years at presentation (OR 2.0,95\% Cl 1.1 to 3.7), presence of chest pain (OR $4.3,95 \% \mathrm{Cl} 2.3$ to 8.1), family history of premature CAD (OR $2.8,95 \% \mathrm{Cl} 1.3$ to 5.9) and utilisation of non-invasive (NIV) stress testing before CAG (OR 3.6, 95\% Cl 1.8 to 7.1). Survival was significantly lower (log rank $p<0.001)$ in patients with $O C A D$ with no revascularisation compared with OCAD with revascularisation or those who had non-obstructive $C A D$, and the latter two groups had comparable survival. Conclusions OCAD is detected in nearly half of the patients with reduced left ventricular systolic function undergoing CAG. Clinical judgement based on thorough history and use of NIV stress testing can help in appropriate patient selection for this test.

\section{INTRODUCTION}

Heart failure with reduced left ventricular ejection fraction (HFrEF) is a common clinical syndrome caused by a variety of cardiac diseases. ${ }^{12}$ Coronary artery disease (CAD) is one of the frequent aetiologies of HFrEF, and the other causes are grouped together as non-ischaemic cardiomyopathy (NICMP). ${ }^{3}$ Diagnosis of ischaemic cardiomyopathy (ICMP) is an independent predictor of mortality, ${ }^{4}$ and the extent of CAD as determined by coronary angiography (CAG) provides incremental prognostic information. ${ }^{5}$ In appropriately selected patients, coronary revascularisation of the ischaemic myocardium can have a favourable prognostic impact. ${ }^{6}$

As per recommendations by current US and European expert guidelines on HF, most US and European cardiac centres perform CAG for the evaluation of patients found to have HFrEF. ${ }^{8}$ The

\section{Key messages}

What is already known about this subject?

- Obstructive coronary artery disease (CAD) is an important and potentially treatable cause of heart failure with reduced left ventricular ejection fraction (HFrEF). Coronary angiography (CAG) is the standard test used to establish CAD as the cause of HFrEF.

- However, CAG is an invasive test with some inherent risk. According to current literature, the frequency of obstructive CAD in patients with HFrEF is variable and depends on patient selection criteria. There are no data from South Asian populations.

What does this study add?

- This study showed that in a selected, real-world, South Asian population of patients referred for cardiac catheterisation, severe obstructive $C A D$ is present in approximately half of the population, similar to previously published data. There is an increased frequency of obstructive $C A D$ in certain patient subsets.

How might this impact on clinical practice?

- Evaluation for CAD should be considered in most patients with a new diagnosis of HFrEF. Utilisation of patient history, CAD risk factors and non-invasive testing remain the mainstay of appropriate patient selection for cardiac catheterisation and CAG.

frequency of obstructive CAD (OCAD) in different studies has varied depending on patient selection criteria, the definition of OCAD and the centre where the study was performed. In a study of select patients who presented at a specialised HF centre with unexplained HF and reduced left ventricular ejection fraction (LVEF), 7\% had OCAD as a major cause for their $\mathrm{HF}^{4}$ while in another study in a general cardiology setting, the incidence of OCAD was found in nearly one-third. ${ }^{9}$

CAG is an invasive test with an inherent risk of complications and can substantially add to healthcare expenditure. Other modalities such as coronary CT angiogram (CCTA), stress echocardiography, myocardial perfusion imaging or cardiac MR image can be reliably used in evaluating OCAD as a cause of HFrEF in carefully selected patients and are potentially less invasive and more economical as well.

There are no published data available on the frequency of OCAD using CAG in patients with HFrEF from South Asia. The primary objective of the study was to determine the incidence of OCAD 
in patients undergoing CAG at a stand-alone cardiology centre with HFrEF as an indication for elective CAG. Finding predictors for OCAD and clinical outcomes in patients with HFrEF was our secondary objective.

\section{METHODS \\ Setting}

The study was performed at Tabba Heart Institute, Karachi, Pakistan. Institutional ethics review board approval was obtained prior to starting the study. A cath-PCI registry, modelled after the US National Cardiac Data Registry (NCDR), has been maintained of all patients undergoing coronary diagnostic or interventional procedures at our institution since May 2012. Patient data were extracted from this registry. Standard NCDR definitions were used for all parameters. HFrEF, as an indicator for elective CAG, is an available parameter in the registry data collection form, defined as patients who have HFrEF (EF 40\% or less on transthoracic echocardiography or nuclear imaging) as the primary indication for CAG.

\section{Patient population and data collection}

Data on all consecutive patients undergoing CAG from May 2012 until July 2016 were reviewed and included in the analysis. We included all outpatients aged more than 18 years of either gender with an EF of $40 \%$ or less who had been referred for CAG.

Patients admitted with acute coronary syndrome, prior myocardial infarction (MI), known CAD, prior percutaneous coronary intervention (PCI) or coronary artery bypass graft $(\mathrm{CABG})$ and having underlying severe primary valvular heart disease or other compelling indications for CAG such as shock or acute pulmonary oedema requiring mechanical ventilation were excluded from the study. LVEF assessed on formal, comprehensive and departmental transthoracic echocardiography was used to quantify LV function.

The key variables included: patient demographics, age, gender, diabetes mellitus, hypertension, smoking history, dyslipidaemia, family history of premature CAD, presence and severity of angina symptoms, history of hospital admission with HF and utilisation of non-invasive (NIV) stress testing prior to CAG. Complications related to angiogram were recorded as a composite of in-hospital death, MI, HF deterioration, major vascular complications or the need for blood transfusion. Follow-up data were obtained from the cath/PCI and CABG registries, medical files were reviewed and, when needed, telephonic follow-ups were taken. The frequencies of patients with HFrEF undergoing PCI or CABG after diagnostic CAG and their indications were recorded. Data on survival status, change in EF and use of essential device therapy as a part of HF management were gathered.

\section{Definition of OCAD}

OCAD was defined as per Felker et al' study of the ischaemic aetiology of $\mathrm{HFrEF},{ }^{10}$ that is, $\geq 75 \%$ stenosis of either the proximal left anterior descending (LAD) or left main coronary artery (LMCA) or $\geq 75 \%$ stenosis of two or more epicardial vessels other than proximal LAD. Coronary stenosis was graded by visual scoring by the interpreting cardiologist at the time of CAG.

\section{Statistical analysis}

SAS V.9.2 was used for statistical analysis. Means and SDs were calculated for continuous variables and percentages were calculated for categorical variables. Incidence of OCAD was reported

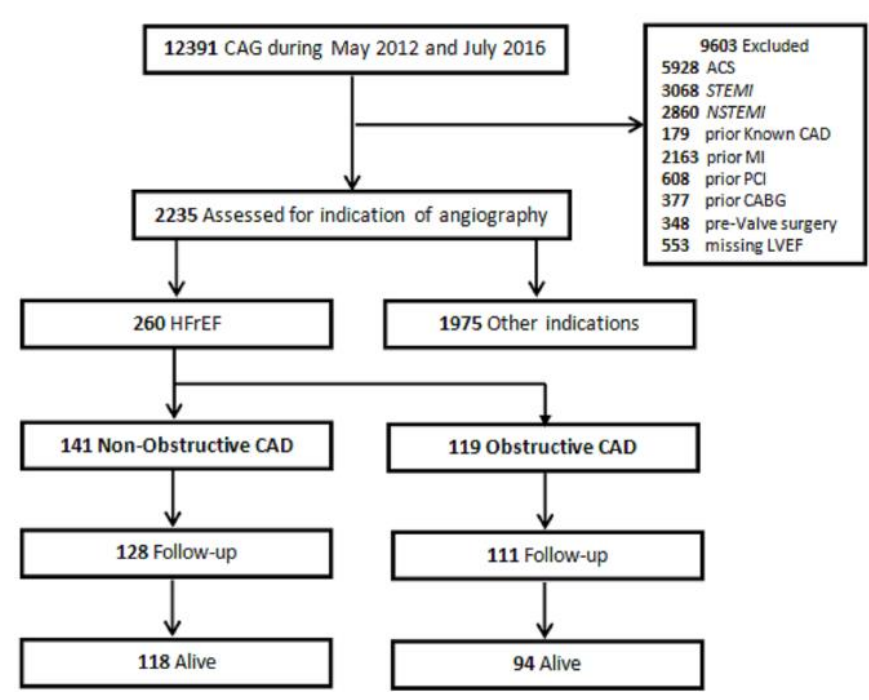

Figure 1 Patient selection during study period. ACS, acute coronary syndrome; $C A B G$, coronary artery bypass graft; $C A D$, coronary artery disease; $C A G$, coronary angiography; $H F r E F$, heart failure with reduced ejection fraction; LVEF, left ventricular ejection fraction; MI, myocardial infarction; NSTEMI, non-ST-elevation myocardial infarction; PCl, percutaneous coronary intervention; STEMI, ST-elevation myocardial infarction.

with percentages. Univariate analysis was performed using $\chi^{2}$ or Fisher's exact test as deemed applicable. Predictors of OCAD were evaluated using a logistic regression model for statistical significance and confounding factors. Variables which were clinically relevant and those with $\mathrm{p}$ values of 0.10 or less in univariable analysis were used for model building and the forward selection method (entry p value of 0.25 and exclusion $p$ value of 0.10 ) was used. ORs with $95 \%$ CIs were reported. Final p values less than 0.05 were considered significant.

Frequencies of complications associated with CAG were also compared using Fisher's exact test. Patients with OCAD who underwent ad hoc PCI were excluded from this comparison. Among those with OCAD, the mode of CAD management was also recorded (revascularised, PCI, CABG or medical management only). Survival was compared at 24 months between OCAD and non-obstructive CAD (NOCAD) groups using Kaplan-Meier curves. Separate survival curves were generated for subgroups in OCAD (revascularised including both PCI and CABG vs medical treatment only). HRs were calculated with 95\% CI.

\section{RESULTS}

During a study period of 4 years, 12391 diagnostic CAGs were performed at our institute. Out of these, 2788 patients met the inclusion criteria. Complete data were available on 2235 (80.1\%) patients, which were included for analysis. The major missing variable was LVEF, which was not available in $20 \%$ of patients and all of these belonged to the group where the indication for CAG was something other than CAD diagnosis in unexplained LV systolic dysfunction. The flow of patients is shown in figure 1.

The overall mean age was $57.6 \pm 9.9$ years. There were 478 $(21.4 \%)$ women in the study. In the majority of patients who underwent elective CAG, the indication for the procedure was a positive NIV stress test (40\%), suspected unstable angina (19.6\%) or evaluation of stable chest pain (28.8\%). Unexplained LV systolic dysfunction was the primary indication for CAG in 
Table 1 Comparison of major variables and univariate analysis among patients undergoing elective coronary angiography (CAG) according to type of indication for the procedure (with or without heart failure with reduced ejection fraction (HFrEF)). $n=2235$

\begin{tabular}{|c|c|c|c|c|c|}
\hline Parameters & $\begin{array}{l}\text { Overall } \\
n=2235\end{array}$ & $\begin{array}{l}\text { HFrEF not a CAG } \\
\text { indication } \\
\mathrm{n}=1975(88.4 \%)\end{array}$ & $\begin{array}{l}\text { HFrEF as CAG } \\
\text { indication } \\
n=260(11.6 \%)\end{array}$ & OR $(95 \% \mathrm{Cl})$ & $P$ value \\
\hline Age (years) & $57.60 \pm 9.9$ & $57.4 \pm 9.6$ & $57.1 \pm 10.5$ & $0.83(0.6$ to 1.13$)$ & 0.08 \\
\hline Females & $478(21.4)$ & $415(21.0)$ & $63(24.2)$ & $0.8(0.6$ to 1.1$)$ & 0.26 \\
\hline Prior heart failure & $81(3.6)$ & $28(1.4)$ & $53(20.4)$ & $17.8(11.0$ to 28.8$)$ & $<0.001$ \\
\hline Smoker & $397(17.8)$ & $357(18.1)$ & $40(15.4)$ & $0.82(0.58$ to 1.2$)$ & 0.30 \\
\hline Dyslipidaemia & $799(35.7)$ & $741(37.5)$ & $58(22.3)$ & $0.48(0.35$ to 0.65$)$ & $<0.001$ \\
\hline Hypertension & $1578(70.6)$ & $1411(71.4)$ & $167(64.2)$ & $0.72(0.55$ to 0.94$)$ & 0.01 \\
\hline Family history of premature CAD & $504(22.6)$ & $461(23.3)$ & $43(16.5)$ & $0.65(0.46$ to 0.92$)$ & 0.007 \\
\hline Diabetes mellitus & $1155(51.7)$ & $1010(51.1)$ & $145(55.8)$ & $1.20(0.93$ to 1.56$)$ & 0.16 \\
\hline History of chest pain & $1539(68.9)$ & $1471(74.5)$ & $68(26.2)$ & $0.12(0.09$ to 0.16$)$ & $<0.001$ \\
\hline Non-invasive stress test & $1025(46.4)$ & $972(49.2)$ & $53(20.4)$ & $0.26(0.19$ to 0.36$)$ & $<0.001$ \\
\hline Positive NIV result (out of total performed) & $937(91.3)$ & $893(91.6)$ & $44(88.2)$ & $0.68(0.33$ to 1.42$)$ & 0.12 \\
\hline Any obstructive CAD* & $1735(77.6)$ & $1613(81.6)$ & $122(46.9) \dagger$ & $0.19(0.15$ to 0.26$)$ & $<0.001$ \\
\hline
\end{tabular}

${ }^{*}$ Any epicardial vessel $>2 \mathrm{~mm}$ with more than $70 \%$ or left main $>50 \%$ stenosis.

†According to Felker et al' definition, obstructive CAD was $45.8 \%$.

CAD, coronary artery disease; NIV, non-invasive.

$11.6 \%(n=260)$ of patients. The mean percentage LVEF in these patients was $27.4 \% \pm 7.4 \%$. There was no significant difference in gender, age, diabetes or smoking history among patients with and without HFrEF. However, significantly more patients with HFrEF had a recent hospitalisation for HF. Patients with HFrEF were less likely to have hypertension, dyslipidaemia or angina symptoms before presentation or NIV stress test prior to CAG. OCAD was less common among patients with HFrEF compared with other CAG indications (table 1).

\section{Primary outcome}

Of the 260 patients undergoing CAG for evaluation of HFrEF, the incidence of OCAD was $45.8 \%(n=119)$. Among patients with OCAD, 71 (59.7\%) had severe proximal LAD disease. Severe LMCA stenosis was found in $4.2 \%$ of these patients while the remaining $36.1 \%$ had two or three vessel obstructive diseases excluding proximal LAD and LMCA. The concomitant involvement of other coronaries in addition to Felker's defined criteria of OCAD is given in figure 2. In the NOCAD group, $107(75.9 \%)$ patients had no obstructive coronary lesion and $24.1 \%$ of patients had an obstructive lesion in one major epicardial vessel (other than proximal LAD and LMCA). Among the HFrEF group, significantly more patients with OCAD had a positive family history of premature CAD and were more likely to have symptoms of chest pain (typical or atypical) compared with patients with NOCAD. Additionally, in the OCAD group, more patients had NIV performed for assessment of ischaemia prior to CAG. There were no significant differences in demographics and other CAD risk factors between the two groups. Detailed unadjusted analysis with ORs and their 95\% CIs is given in table 2 .

\section{Secondary outcome}

In multivariate analysis, major independent predictors of OCAD on CAG were: age above 50 years at presentation (OR 2.0, 95\% CI 1.1 to 3.7), presence of chest pain (OR 4.3, 95\% CI 2.3 to 8.1), family history of premature CAD (OR 2.8, 95\% CI 1.3 to 5.9) and NIV stress testing before CAG (OR 3.6, 95\% CI 1.8 to 7.1 ) (details in table 3 , all $\mathrm{p}$ values were $<0.05$ ). Diabetes mellitus with borderline significance in univariate analysis was statistically non-significant on multivariate analysis $(p=0.14)$. There was no significant interaction among the predictors.

There was one in-hospital death in the NOCAD group after CAG secondary to progressive pump failure while no complications of CAG were seen in the OCAD group. Treatment recommendations were decided primarily by the treating cardiologist and cardiac surgeons in a heart team approach. Among the patients with OCAD, 67 (56.3\%) were recommended for CABG and $18(15.1 \%)$ were recommended for PCI by their treating cardiologist. Of these patients, 61 (23.1\% overall; $52.5 \%$ of OCAD) underwent revascularisation (CABG: 46, PCI: 15). The remaining $47.5 \%$ (58) of these patients did not undergo

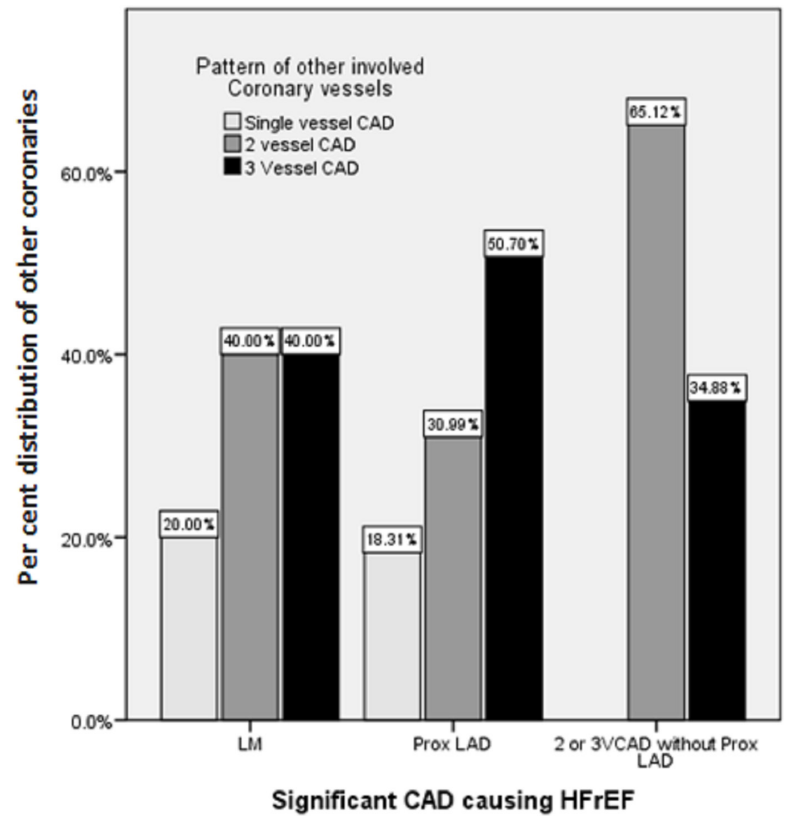

Figure 2 Pattern of other coronary lesions in obstructive CAD group other than Felker's defined criteria of $200 \times 179 \mathrm{~mm}(300 \times 300$ dots per inch (DPI)). CAD, coronary artery disease; $\mathrm{HFrEF}$, heart failure with reduced ejection fraction; $L A D$, left anterior descending; $L M$, left main coronary artery; VCAD, vessel CAD. 
Table 2 Characteristics of patients undergoing coronary angiography (CAG) with heart failure with reduced ejection fraction (HFrEF) and univariable analysis of obstructive coronary artery disease (OCAD) versus non-obstructive coronary artery disease (NOCAD) ( $n=260)$

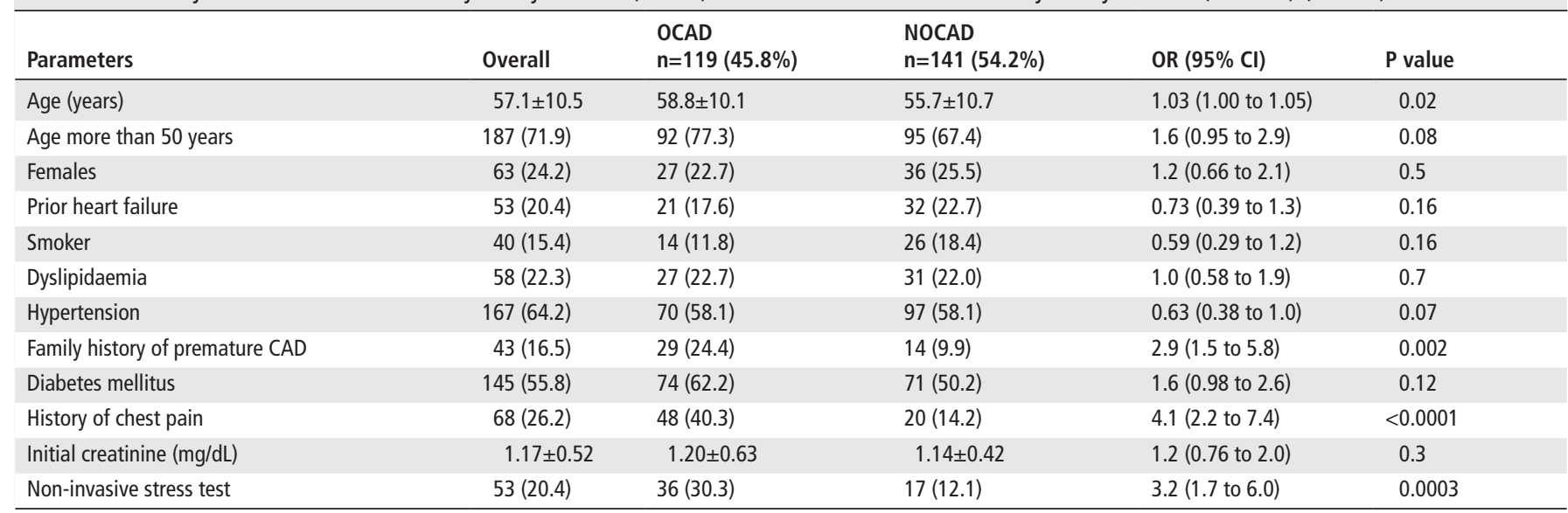

CAD, coronary artery disease.

revascularisation. The reasons for not performing revascularisation in these patients were as follows: anatomy not favourable for PCI or CABG in 18 (15.1\% of OCAD), medical therapy advised in 11 (9.2\%) due to the absence of ischaemia or viability on functional testing and no reason was documented in 8 (6.7\%). A total of $21(17.6 \%)$ patients declined revascularisation and opted for guideline-directed medical therapy (GDMT). Details on revascularisation recommendations and actual treatment are displayed in figure 3. Details on GDMT are provided in table 4.

\section{Follow-up events}

Follow-up was available for 239 (92\%) patients for a median duration of 25 months (IQR: 22 months). There were 27 $(11.3 \%)$ deaths in total. There was a statistically significant difference in survival among NOCAD versus OCAD (92.2\% vs $84.7 \%$, respectively, log rank test $\mathrm{p}$ value 0.02 , HR: $1.1-5.3)$. When survival status was compared between all patients with NOCAD and OCAD subgroups based on their revascularisation status, the difference in survival was mostly driven by whether OCAD was revascularised or not (figure 4). The survival as shown in table 5 was similar between patients with OCAD who underwent revascularisation and patients with NOCAD (93.1\% vs $92.2 \%$, HR $1.0 ; 95 \%$ CI 0.3 to 3.1 ), while patients with OCAD who were not revascularised had significantly lower survival compared with patients with OCAD who were revascularised (93.1\% vs $75.5 \%$; HR 4.3, 95\% CI 1.4 to 13.2 ).

There was no significant difference in baseline LVEF among OCAD with and without revascularisation; however, there was significant improvement in LVEF in 38 out of 61 revascularised patients who had their echocardiogram performed at a mean follow-up of 12 months after revascularisation $(29.7 \% \pm 6.6 \%$ vs $38.9 \% \pm 8.5 \%, \mathrm{p}<0.0001)$. In multivariate

Table 3 Predictors of obstructive CAD on multivariable regression analysis in patients undergoing CAG with heart failure with reduced ejection fraction (HFrEF), $n=260$. ORs and $95 \% \mathrm{Cls}$ are shown

\begin{tabular}{llll}
\hline & Adjusted ORs & O5\% Cl for & \\
& OR & Significance \\
\hline Presence of angina symptoms & 4.3 & 2.3 to 8.1 & $<0.00001$ \\
Non-invasive testing performed & 3.6 & 1.8 to 7.1 & 0.0002 \\
Family history of premature CAD & 2.8 & 1.3 to 5.9 & 0.007 \\
\hline Age more than 50 years & 2.0 & 1.1 to 3.7 & 0.03 \\
\hline
\end{tabular}

CAD, coronary artery disease; CAG, coronary angiography. analysis of independent predictors of mortality in the OCAD group according to revascularisation status, the only significant mortality predictor in the non-revascularised subgroup was presence of severe proximal LAD lesion regardless of concomitant involvement of other coronary arteries $\left(\chi^{2}\right.$ value: 5.99, $\mathrm{p}=0.049$ ). Cardiac resynchronisation therapy (CRT) or implantable cardioverter-defibrillator (ICD) was performed in five patients during follow-up, of which four patients were inserted in the NOCAD group and one in the OCAD group.

\section{DISCUSSION}

In this study, we found that the incidence of OCAD was $46 \%$ in 260 patients with HFrEF without prior CAD who underwent CAG as part of workup for HFrEF evaluation. The independent predictors of OCAD are age more than 50 years, history of chest pain, family history of premature CAD and abnormal NIV ischaemia testing. Approximately half of the patients with OCAD underwent revascularisation and had improvement in LV function and survival compared with patients who were not revascularised. The patients with NOCAD also appeared

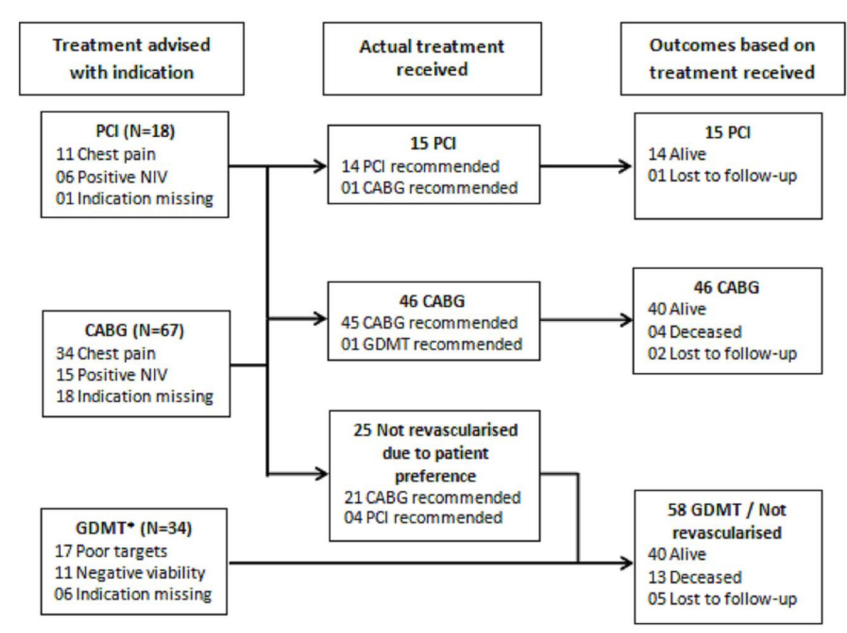

* Guideline directed medical therapy

Figure 3 Flow chart of management of patients with obstructive coronary artery disease (OCAD) according to revascularisation strategy. CABG, coronary artery bypass graft; NIV, non-invasive; $\mathrm{PCl}$, percutaneous coronary intervention. 
Table 4 Guideline-directed medical therapy (GDMT) at the time of discharge in patients undergoing coronary angiography (CAG) with heart failure with reduced ejection fraction (HFrEF)

\begin{tabular}{|c|c|c|c|c|c|}
\hline Parameters & Overall & $\begin{array}{l}\text { OCAD } \\
n=119(45.8 \%)\end{array}$ & $\begin{array}{l}\text { NOCAD } \\
n=141(54.2 \%)\end{array}$ & OR $(95 \% \mathrm{Cl})$ & $P$ value \\
\hline Antiplatelet medications & $213(81.9)$ & $114(95.8)$ & $99(70.2)$ & 9.6 (3.7 to 25.1$)$ & $<0.0001$ \\
\hline ACE-I or ARBs & $205(78.8)$ & $93(71.2)$ & $112(79.4)$ & $0.92(0.51$ to 1.7$)$ & $N S^{*}$ \\
\hline Beta-blockers & $231(88.8)$ & $104(87.4)$ & $137(90.1)$ & $0.76(0.35$ to 1.6$)$ & NS* \\
\hline Aldosterone blocking agents & $117(45.0)$ & $49(41.2)$ & $58(48.2)$ & $0.75(0.45$ to 1.2$)$ & NS* \\
\hline Diuretics & $140(53.8)$ & $55(46.2)$ & $85(60.3)$ & $0.56(0.34$ to 0.92$)$ & 0.02 \\
\hline
\end{tabular}

${ }^{*} \mathrm{P}>0.10$.

$A C E-I, A C E$ inhibitor; ARB, angiotensin receptor blocker; NOCAD, non-obstructive coronary artery disease; NS, not significant; OCAD, obstructive coronary artery disease.

to have favourable prognosis compared with non-revascularised OCAD.

In two studies from Brazil and Europe, OCAD is the main cause of HFrEF, accounting for approximately $32 \%$ of cases of HFrEF. ${ }^{11} 12$ Bart et al showed an OCAD prevalence of $93 \%$ in patients with HF. ${ }^{13}$ The results of our study lie between the above two. This variability is mostly due to use of different definitions for significant CAD and difference in patient populations. We used the Felker's definition of OCAD, as it implies a greater ischaemic burden with more likelihood that the OCAD is the underlying cause of HFrEF rather than an incidental finding.

Most prior studies have shown that established coronary risk factors differ between ICMP and NICMP ${ }^{14-16}$; however, in our study most of the clinical parameters were equally distributed among the two groups except for age, chest pain history and family history of CAD. This perhaps is related to selection bias, with patients with a lower likelihood of CAD not being referred for CAG. In a study ${ }^{14}$ performed on the Duke Database of approximately 2000 patients who underwent CAG to assess ischaemic aetiology, typical angina was present in approximately $40 \%$ of patients classified as NICMP, while in our study this proportion with any chest pain, either typical or atypical, was only $14 \%$ compared with $43 \%$ in the OCAD group.

Cardiac catheterisation in patients with dilated cardiomyopathy or with symptoms of HF is associated with a 2.2-3.3 times higher risk of complications compared with an unselected population $(1.7 \%) .{ }^{17}{ }^{18}$ In the study by Laskey et al, the incremental benefit was less favourable, as procedure-related complications were $2.8 \%$ and only $3.7 \%$ of patients subsequently underwent revascularisation. ${ }^{19}$ In our study, the risk of adverse complications related to CAG was very low

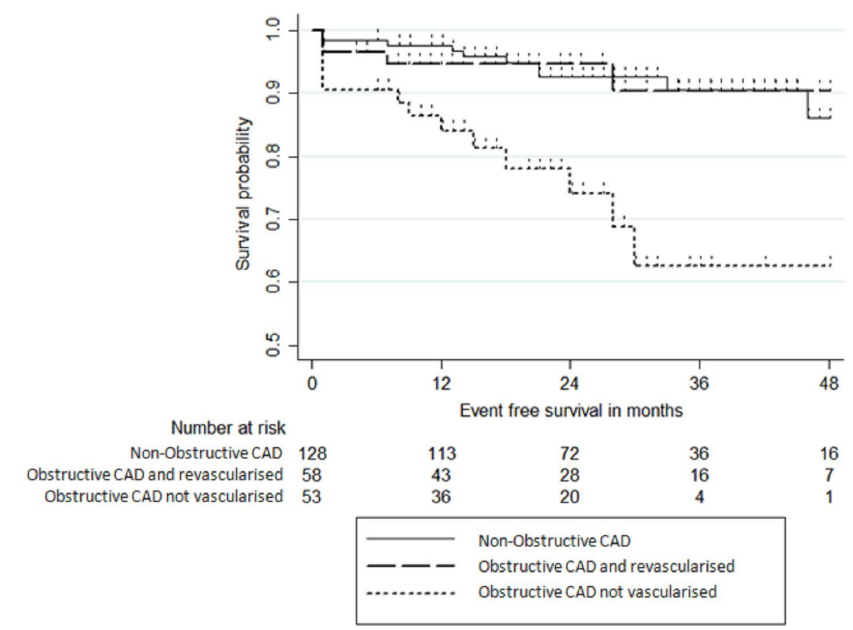

Figure 4 Kaplan-Meier survival analysis. CAD, coronary artery disease.
$(0.5 \%)$, with a much higher subsequent revascularisation rate. This is likely due to $>95 \%$ use of radial artery access and a more selected patient population. NIV evaluation using either coronary artery calcium (CAC) scoring only or CCTA may be another reliable and potentially lower risk means of excluding OCAD as a cause of HFrEF. ${ }^{20-22}$

Prior studies also suggest that there is potential reversibility of HFrEF and improved survival in patients with CAD and HFrEF when revascularisation is performed, especially when guided by viability testing. ${ }^{17}$ A meta-analysis by Allman et al demonstrated improvement in LV function and survival after revascularisation and its association with myocardial viability on NIV testing. ${ }^{6}$ The only randomised trial of revascularisation in HFrEF, the STICH (Surgical Treatment for Ischemic Heart Failure) trial, showed an improvement in survival and repeat hospitalisation rates at 9.8 years' follow-up when patients were revascularised by coronary bypass grafting compared with medical therapy. ${ }^{18}$ In our study, in patients with HFrEF and OCAD, the prognosis appeared to be better after revascularisation and the improvement in outcome manifested earlier compared with the STICH trial despite being a much smaller population. This is likely due to a selection bias with patients referred for revascularisation being younger in age with more favourable coronary anatomy, greater frequency of angina symptoms or myocardial viability on ischaemia testing and less advanced adverse cardiac remodelling .

Despite their proven benefits from randomised trials in terms of mortality and quality of life in both NOCAD and OCAD, ${ }^{20} 23$ utilisation of devices (ICD and CRT) remains suboptimal in the real world. ${ }^{21} 22$ The findings of our study are consistent with prior results and are also a reflection of medical practice in a severely resource-constrained economic environment.

Published studies comparing survival in patients with HFrEF based on whether ischaemia is the cause for cardiomyopathy have shown variable results. Some studies show similar prognosis in such patients ${ }^{24}$ while a US-based study showed that ICMP has

Table 5 Cox proportional hazards model for comparison of survival among non-obstructive $C A D$ and obstructive $C A D$ with and without revascularisation in patients with heart failure with reduced ejection fraction (HFrEF) $(n=239)$. HRs and $95 \% \mathrm{Cls}$ are shown

\begin{tabular}{llll}
\hline & $\begin{array}{l}\text { Survival } \\
\text { rate }(\%)\end{array}$ & HRs $(95 \% \mathrm{CI})$ & Significance \\
\hline $\begin{array}{l}\text { Obstructive CAD with } \\
\text { revascularisation }\end{array}$ & 93.1 & Reference & - \\
$\begin{array}{l}\text { Non-obstructive CAD } \\
\begin{array}{l}\text { Obstructive CAD without } \\
\text { revascularisation }\end{array}\end{array}$ & 92.2 & $1.0(0.3$ to 3.1) & NS* \\
\hline $\begin{array}{l}\text { *P } P \text { 0.05. } \\
C A D \text {, coronary artery disease; NS, not significant. }\end{array}$ & $4.3(1.4$ to 13.2) & 0.01 \\
\hline
\end{tabular}


a worse prognosis. ${ }^{25}$ A recent randomised trial of patients with NICMP comparing ICD therapy to no ICD showed greater than $90 \%$ survival at 2 years in both groups. ${ }^{22}$ The NOCAD group in our study showed similar ( $>90 \%$ survival) at 2 years. Based on this, the difference in outcome between the OCAD and NOCAD groups in our study does suggest a more favourable outcome in patients with NICMP.

According to the current management guidelines for HF, CAG should be considered in the diagnostic evaluation of patients with HFrEF in the presence of risk factors for CAD, angina or refractory symptoms of HF. ${ }^{78}$ Our findings reinforce these recommendations, as symptoms of angina and presence of risk factors are predictors of OCAD. In our study population, in patients younger than 50 years of age with no chest pain, no family history of premature CAD and no NIV stress testing, the likelihood of OCAD is only 10\% (4 out of 36). Therefore, more selective performance of CAG in patients with HFrEF will improve diagnostic yield without compromising patient care and will also be more cost-effective. Additionally, the use of less invasive and possibly more economical tests such as CCTA and CAC score can work as a gatekeeper.

The major limitations of our study are that it is a retrospective single-centre study that may have an element of measurement bias. Inclusion was based on documentation in the database listing HFrEF as the primary indication for CAG by the data collector, which may not be accurate. The study population comprised selected patients referred for CAG by the treating cardiologist and patients with very low risk for CAD who might not have been advised to undergo CAG. This may induce a referral bias that would inherently result in overestimation of OCAD in patients with HFrEF. No invasive evaluation was performed to assess the physiological significance of intermediate-grade lesion $(50 \%-70 \%)$ stenosis using fractional flow reserve due to low utilisation of this modality in our retrospective data. Finally, all-cause mortality was chosen as the main outcome as an alternative of cardiac mortality due to the absence of national-level mortality registries. Follow-up was only missing in a small proportion of patients.

\section{CONCLUSION}

OCAD is present in a significant proportion of patients undergoing CAG for evaluation of CAD in patients with HFrEF. The likelihood of OCAD detection is higher in patients with a history of chest pain, older age or abnormal NIV ischaemia testing. Survival appears to be more favourable in patients who undergo revascularisation and among patients with NOCAD.

Acknowledgements We acknowledge the contributions from our faculty and fellows in helping to maintain the NCDR-based cath-PCI registry in our institute, which is the source of data for this study. We are also grateful to our research team including Dr Wahaj Fatmi, Shamsa Nadeem, Shumaila Tarani and Zubair Khan for their support in data collection.

Contributors All authors were directly responsible for generating the research idea, designing the study, analysis and interpretation of the results and drafting and revising of the manuscript. The submitted version was finalised through mutual discussion and agreement. In addition, the acknowledged contributors have agreed to the inclusion of their names.

Funding The authors have not declared a specific grant for this research from any funding agency in the public, commercial or not-for-profit sectors.

Competing interests None declared.

Patient consent for publication Not required.
Ethics approval Ethical review board of Tabba Heart Institute.

Provenance and peer review Not commissioned; externally peer reviewed.

\section{REFERENCES}

1. Malaquin D, Tribouilloy C. [Epidemiology of heart failure]. Rev Prat 2010;60:911-5.

2. Gheorghiade M, Bonow RO. Chronic heart failure in the United States. Circulation 1998:97:282-9.

3. McCrohon JA, Moon JCC, Prasad SK, et al. Differentiation of heart failure related to dilated cardiomyopathy and coronary artery disease using gadolinium-enhanced cardiovascular magnetic resonance. Circulation 2003;108:54-9.

4. Felker GM, Thompson RE, Hare JM, et al. Underlying causes and long-term survival in patients with initially unexplained cardiomyopathy. $N$ Eng/ I Med Overseas Ed 2000;342:1077-84.

5. Burggraf GW, Parker JO. Prognosis in coronary artery disease. angiographic, hemodynamic, and clinical factors. Circulation 1975;51:146-56.

6. Allman KC, Shaw LJ, Hachamovitch R, et al. Myocardial viability testing and impact of revascularization on prognosis in patients with coronary artery disease and left ventricular dysfunction: a meta-analysis. J Am Coll Cardiol 2002;39:1151-8.

7. Yancy CW, Jessup M, et al. 2013 ACCF/AHA guideline for the management of heart failure. Circulation 2013;62.

8. McMurray JJ, Adamopoulos S, Anker SD, et al. ESC guidelines for the diagnosis and treatment of acute and chronic heart failure 2012: the task Force for the diagnosis and treatment of acute and chronic heart failure 2012 of the European Society of cardiology. developed in collaboration with the heart Failure Association (HFA) of the ESC. Euro J Heart Fail 2012;33:1787-847.

9. Soriano CJ, Ridocci F, Estornell J, et al. Noninvasive diagnosis of coronary artery disease in patients with heart failure and systolic dysfunction of uncertain etiology, using late gadolinium-enhanced cardiovascular magnetic resonance. J Am Coll Cardiol 2005:45:743-8.

10. Felker GM, Shaw LK, O'Connor CM. A standardized definition of ischemic cardiomyopathy for use in clinical research. J Am Coll Cardiol 2002;39:210-8.

11. Melo RMVde, Melo EFPde, Biselli B, et al. Clinical usefulness of coronary angiography in patients with left ventricular dysfunction. Arq Bras Cardiol 2012;98:437-41.

12. Silva F, Borges $T$, Ribeiro $A$, et al. Heart failure with reduced ejection fraction: should we submit patients without angina to coronary angiography? Int I Cardiol 2015:190:131-2.

13. Bart $B A$, Shaw $L K, M c C$ ants $C B$, et al. Clinical determinants of mortality in patients with angiographically diagnosed ischemic or nonischemic cardiomyopathy. J Am Coll Cardiol 1997;30:1002-8.

14. Whellan DJ, Tuttle RH, Velazquez EJ, et al. Predicting significant coronary artery disease in patients with left ventricular dysfunction. American Heart Journal 2006;152:340-7.

15. Steg PG, Dabbous $\mathrm{OH}$, Feldman $\mathrm{L}$, et al. Determinants and prognostic impact of heart failure complicating acute coronary syndromes. Circulation 2004;109:494-9.

16. Doukky R, Shih MJ, Rahaby M, et al. A simple validated clinical tool to predict the absence of coronary artery disease in patients with systolic heart failure of unclear etiology. Am J Cardiol 2013;112:1165-70.

17. Bax JJ, Poldermans D, Elhendy A, et al. Improvement of left ventricular ejection fraction, heart failure symptoms and prognosis after revascularization in patients with chronic coronary artery disease and viable myocardium detected by dobutamine Stress Echocardiography. J Am Coll Cardiol 1999;34:163-9.

18. Velazquez $\mathrm{EJ}$, Lee $\mathrm{KL}$, Jones $\mathrm{RH}$, et al. Coronary-artery bypass surgery in patients with ischemic cardiomyopathy. N Engl J Med 2016;374:1511-20.

19. Laskey W, Boyle J, Johnson LW. Multivariable model for prediction of risk of significant complication during diagnostic cardiac catheterisation. Catheterisation and Cardiovascular Interventions 1993;30:185-90.

20. Wasmer K, Köbe J, Andresen D, et al. Comparing outcome of patients with coronary artery disease and dilated cardiomyopathy in ICD and crt recipients: data from the German DEVICE-registry. Clin Res Cardiol 2013;102:513-21.

21. Chia P-L, Foo D. Overview of implantable cardioverter defibrillator and cardiac resynchronisation therapy in heart failure management. Singapore Med J 2016:57:354-9.

22. van Veldhuisen DJ, Maass AH, Priori SG, et al. Implementation of device therapy (cardiac resynchronization therapy and implantable cardioverter defibrillator) for patients with heart failure in Europe: changes from 2004 to 2008. Eur J Heart Fail 2009;11:1143-51.

23. Lindenfeld J, Feldman AM, Saxon L, et al. Effects of cardiac resynchronization therapy with or without a defibrillator on survival and hospitalizations in patients with new York Heart Association class IV heart failure. Circulation 2007;115:204-12.

24. Lourenço C, Saraiva F, Martins H, et al. Ischemic versus non-ischemic cardiomyopathy-are there differences in prognosis? Experience of an advanced heart failure center. Rev Port Cardiol 2011:30:181-97.

25. Gajanana D, Shah M, Junpapart P, et al. Mortality in systolic heart failure revisited: ischemic versus non-ischemic cardiomyopathy. Int I Cardiol 2016;224:15-17. 